\title{
Characterization of Surface Variation of Chemically Amplified Photoresist to Evaluate Extreme Ultraviolet Lithography Stochastics Effects
}

\author{
Eric Liu $^{{ }^{*},}$, Amir Hegazy ${ }^{1}$, Hyeonseon Choi ${ }^{1}$, Maximilian Weires ${ }^{1}$ \\ Robert Brainard ${ }^{1}$, and Gregory Denbeaux ${ }^{1}$ \\ ${ }^{1}$ College of Nanoscale Science and Engineering, State University of New York \\ Polytechnic Institute, 257 Fuller Road, Albany, NY, 12203, USA \\ *eliu@sunypoly.edu
}

\begin{abstract}
Extreme ultraviolet (EUV) lithography is required for advanced node semiconductor device fabrication. The stochastic effects in EUV lithography are problematic, especially with regards to pattern roughness and defect formation. In this study, we performed atomic force microscopy (AFM) on an EUV photoresist surface to determine the surface roughness, height histogram, line scan, area ratio, and power spectral density (PSD). Polymethyl methacrylate (PMMA) for nonchemically amplified resist (non-CAR), and poly(4-hydroxystyrene)(t-butyl acrylate) copolymer (PHS:tBA) and poly(4-hydroxystyrene)(polystyrene)(t-butyl acrylate) copolymer (PHSPS:tBA) with/ di-(t-butylphenyl)iodonium perfluorobutane sulfonate (TBPI-PFBS)/tetrabutylammonium lactate (TBAL) for chemically amplified resist (CAR) were examined. In this CAR system, the exposure and dark loss contributed to the surface variation of root mean square (RMS) of $1.5 \mathrm{~nm}$ and $0.95 \mathrm{~nm}$ under a nominal exposure dosage of $8 \mu \mathrm{C} / \mathrm{cm} 2$. The contribution of dark loss was further evaluated from the effects of backbone polymer composition and photoacid generator (PAG) loading. The dark loss induced surface roughness can be attributed to the competition of etch selectivity in the resist components. A skewness of the height histogram and change of correlation in PSD are related to the dark loss induced surface variation.
\end{abstract}

Keywords: EUV, Stochastics effects, AFM, CAR, Non-CAR, Surface roughness.

\section{Introduction}

In the semiconductor industry, device dimension reduction is one of the most effective strategies to reduce the manufacturing cost and enhance the device performance. A lithography process, known as extreme ultraviolet (EUV) lithography, has been successfully introduced into the advanced logic node device fabrication. Given the novelty of EUV lithography, still several areas require extensive research efforts such as productivity, pattern fidelity, and stochastic effects [1]-[4]. In general, the stochastic effects in EUV lithography include the contributions from the probability distribution between photons, electrons, the photoresist material, and post-exposure treatments [5], [6]. These stochastic effects usually form defects in the devices and introduce pattern variations in the physical features [2], [7]-[10]. The pattern variation is generally described as line-edge-roughness (LER) in line/space patterns and contact-edge-roughness (CER) in circular patterns. High LER and CER in semiconductor logic devices deteriorate the device performance [11]-[15].

In EUV lithography, the use of photoresist can be categorized into two types: CAR and Non-CAR. CAR generally contains the backbone polymers, PAG, and a base, known as the quencher. The imaging mechanism and reaction of CAR have been well studied in the past [16]-[18]. The complexity of resist material, chemically amplified reaction, and EUV lithography exposure conditions make the estimation of stochastic effects and physical variation in CAR a complex task. To understand the surface variation of photoresist, one of the most common physical characterization methods is AFM. AFM has been widely used for the characterization 
of polymer surfaces as well as the EUV photoresists in the past few decades. Several research groups have shown a correlation of surface roughness obtained by AFM to the lithography condition and photoresist type [19]-[26]. The previous study reported an elevated surface roughness in the CAR, which was correlated with dark loss induced surface roughness and resist composition [21]. Another study correlated the surface roughness with device yield and defectivity in the CAR [26]. Most AFM data in previous studies showed just the RMS for the surface roughness. A more detailed data processing can be carried out to construct a complete physical presentation of the photoresist surface such as power spectral density (PSD), area ratio, and histogram of height distribution.

In this study, AFM was used to characterize the surface of photoresist as shown in Fig. 1. The data was then used in the next step of data processing to determine the histogram, area ratio/ particle counts, and PSD. The height distribution, occupied area of peak/valley, and correlation length between two equivalent height signals within the scan area were also extracted. All this information was used to decouple the source of variation from each element in both non-CAR and CAR and to understand the EUV lithography stochastic effects as well as the inhomogeneity behavior of development-induced surface variation.

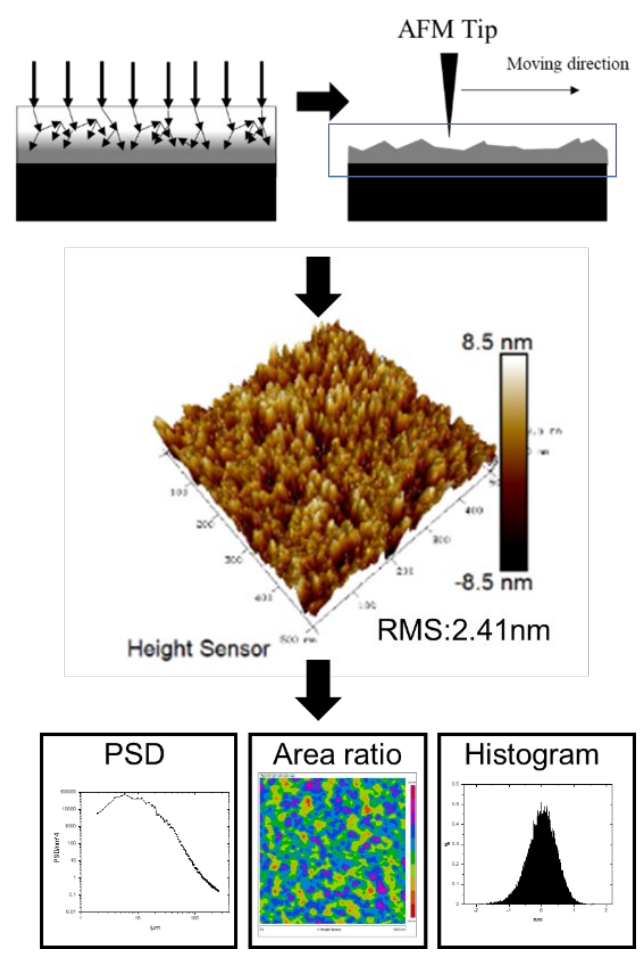

Fig. 1. Illustration of AFM data collection on photoresist surface and data processing.

\section{Experimental}

\subsection{Sample preparation}

The CAR was synthesized using $2 \mathrm{wt} \%$ of a solid polymer dissolved in propylene glycol methyl ether acetate (PGMEA). The solid polymer is a combination of poly(4-hydroxystyrene) (t-butyl acrylate) copolymer (PHS:tBA) or poly(4hydroxystyrene)(polystyrene)(t-butyl acrylate) copolymer (PHSPS:tBA) as the backbone polymer, with di-(t-butylphenyl)iodonium perfluorobutane sulfonate (TBPI-PFBS) as the PAG, and tetrabutylammonium lactate (TBAL) as the quencher. PHS:tBA copolymer has a composition ratio of 60:40 and an average molecular weight of $11,500 \mathrm{~g} / \mathrm{mol}$. The two types of copolymers PHSPS:tBA, PHSPS:tBA 074 with a composition ratio of $61: 24: 15$ and PHSPS:tBA 075 with a composition ratio of $66: 19: 15$, were obtained from Dow Chemical. The nominal condition of CAR in this study is $83.5 \mathrm{wt} \%$ of PHS:tBA or PHSPS:tBA polymer, $15 \mathrm{wt} \%$ of TBPI-PFBS as PAG, and 1.5 $\mathrm{wt} \%$ of TBAL as base. The non-CAR was obtained as a premade $950 \mathrm{k}$ molecular weight PMMA from MicroChem. The chemical structures of both CAR and non-CAR are shown in Fig. 2.

The photoresist was spin-coated onto 4-inch silicon substrates. The spin coat conditions for the CAR are $5 \mathrm{~s}$ in $500 \mathrm{rpm}$, followed by $45 \mathrm{~s}$ in 2000 $\mathrm{rpm}, 5 \mathrm{~s}$ in $500 \mathrm{rpm}$, and $60 \mathrm{~s}$ in $1500 \mathrm{rpm}$ for nonCAR. The wafer was subjected to a post-apply bake (PAB) on a hot plate at $120^{\circ} \mathrm{C}$ for $60 \mathrm{~s}$ for the CAR and $180{ }^{\circ} \mathrm{C}$ for $75 \mathrm{~s}$ for the non-CAR. The samples were exposed to an electron beam (e-beam) in an Electron-Resist Interaction Chamber (ERIC) at the College of Nanoscale Science and Engineering in Albany, NY, described elsewhere previously [27][30]. The e-beam energy was $80 \mathrm{eV}$, and the nominal dosage of $8 \mu \mathrm{C} / \mathrm{cm} 2$. After the e-beam exposure, the samples were treated by PEB on a hot plate at 120 ${ }^{\circ} \mathrm{C}$ for $90 \mathrm{~s}$. The CAR samples were rinsed with the developer solution consisting of $0.13 \mathrm{~N} / 0.26 \mathrm{~N}$ tetramethylammonium hydroxide (TMAH) at room temperature for $45 \mathrm{~s}$, followed by $30 \mathrm{~s}$ of deionized water rinse and drying with nitrogen. For the nonCAR samples, the samples were developed in 1:3 methyl isobutyl ketone/isopropyl alcohol for $30 \mathrm{~s}$ at room temperature, followed by deionized water rinse and nitrogen drying. The development-only samples were coated and baked under the PAB conditions. The tested development conditions included a development time from $0 \mathrm{~s}$ to $110 \mathrm{~s}$. 


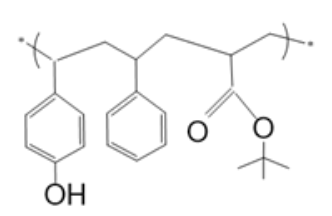

(a)

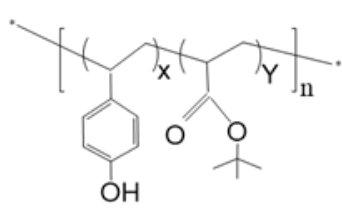

(b)

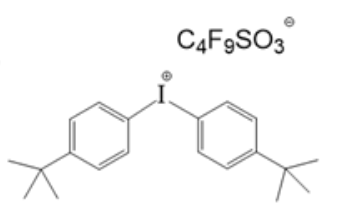

(c)

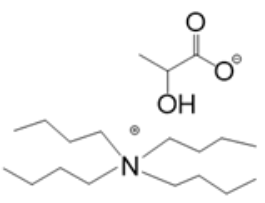

(d)

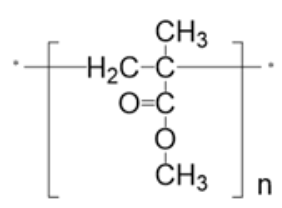

(e)

Fig. 2. Chemical structure of CAR (a) PHSPS:tBA, (b) PHS:tBA, (3) TBPI-PFBS, and (d) TBAL and nonCAR (e) PMMA.

\subsection{Metrology}

The surface roughness was characterized using a Dimension Icon AFM system from Bruker. The measurements were conducted in the noncontact mode of SCANASYST-AIR scan. The tip was made of a triangular shaped silicon nitride with $2 \mathrm{~nm}$ tip radius. The scan area was $0.25 \mu \mathrm{m}^{2}$. The horizontal scan speed was maintained at $500 \mathrm{~nm}$ per second, and the step size in vertical direction was $<4 \mathrm{~nm}$ per step. The results were processed using a NanoScope Analysis software from Bruker using a third-order flattening and noise removal filter to obtain the RMS, PSD, and height distribution histogram by centering the highest point. The AFM images were converted into a quantized color contour image. The area ratio was calculated from a feature with an area of $6 \mathrm{~nm}$ and below by normalizing the highest height of each data point. The thickness loss was obtained using a J.A. Woollam M-2000V spectroscopic ellipsometer equipped with a Cauchy thickness model for photoresist on the silicon substrate.

\section{Results and discussion}

3.1. Surface roughness of PHSPS:tBA CAR vs PMMA non-CAR

To separate different sources of surface roughness on the resist, a simplified model was used assuming that each process is an independent source of roughness. Under the assumption of independent parameter, this simple view of EUV lithography stochastic effects on surface roughness is shown as Equations (1) and (2) for the non-CAR and CAR cases

$$
\begin{aligned}
& \sigma_{\text {Total of Non-CAR }}^{2}=\sigma_{\text {Coat }}^{2}+\sigma_{\text {Exp }}^{2}+\sigma_{\text {Dev }}^{2} \\
& \sigma_{\text {Total of CAR }}^{2}=\sigma_{\text {Coat }}^{2}+\sigma_{\text {Exp }}^{2}+\sigma_{\text {Dev }}^{2}
\end{aligned}
$$

$\sigma_{\text {Total of Non-CAR }}$ and $\sigma_{\text {Total of CAR }}$ are the photoresist surface roughness in non-CAR and CAR, respectively. $\sigma_{\text {Coat }}$ is the surface roughness after coating, representing the surface roughness before exposure. This includes the contributions from resist conformity, uniformity, and film adhesion to the substrate. $\sigma_{\text {Dev }}$ is the surface roughness after the development process includes dark loss induced deprotection reaction variations in the developing process. $\sigma_{\text {Exp }}$ is the surface roughness from the exposure step. This represents the contributions from shot noise and chemical amplified reaction in CAR. This embodies the contributions from material inhomogeneity and Poisson probability distribution in the chemical amplified reaction. This includes photon- electron interaction from the incoming photons, electron cascade within the photoresist material, acid quantum yield, acid diffusion, acid-base neutralization, and the degree of deprotection reaction.

The RMS of surface roughness was collected on both the PMMA as the non-CAR sample and the PHSPS:tBA+TBPI-PFBS+TBAL as the CAR sample in each processing step. The AFM images of both topview and tilt view for the non-CAR and CAR surfaces are shown in Fig. 3. The RMS of both samples significantly decreased after coating in the following processes. In the non-CAR material, dark loss contributed to the RMS degradation from $0.4 \mathrm{~nm}$ to $0.5 \mathrm{~nm}$. Further degradation to $0.8 \mathrm{~nm}$ can be explained by a combination of dark loss and ebeam exposure. Using Equation 1, the $\sigma_{\text {Exp }}$ was calculated as $0.63 \mathrm{~nm}$ in RMS to the overall roughness. In the CAR, the dark loss contributed to the RMS of $0.95 \mathrm{~nm}$. The RMS roughness further degraded to $1.8 \mathrm{~nm}$ due to the contributions from the exposure and chemical amplified reaction. Using Equation 2, the contribution of $\sigma_{\operatorname{Exp}}$ was calculated as $1.5 \mathrm{~nm}$ in RMS. The contribution of each term is shown in Fig. 4. In the CAR system, the exposure and dark loss can be attributed to the major portion of overall surface variation in this formulation. 


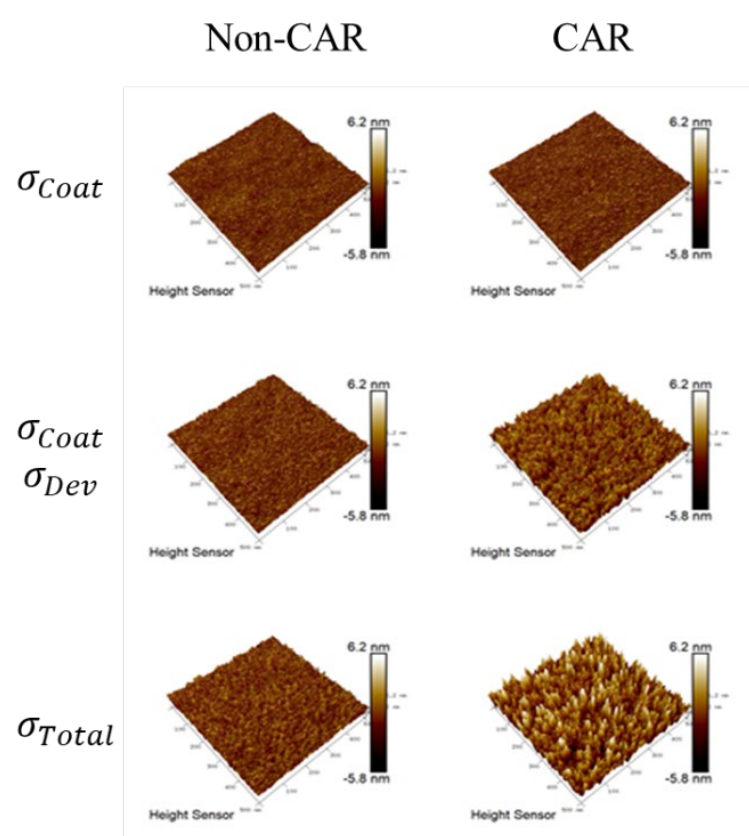

Fig. 3. AFM images of resist surface of PMMA and PHSPS:tBA+TBPI-PFBS+TBAL

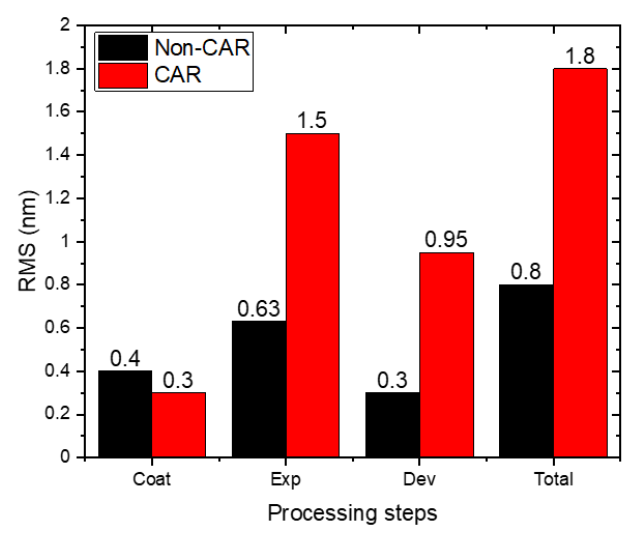

Fig. 4. Contribution of PMMA (Non-CAR) and PHSPS:tBA+TBPI-PFBS+TBAL (CAR) to surface roughness.

\subsection{Dark loss induced surface variation in PHS:tBA system}

In the following sections, the dark loss induced contribution of resist compositions to surface roughness is discussed, especially the effect of polymer system and PAG loading. The first evaluated CAR sample was PHS:tBA as the backbone polymer. Fig. 5 (a) shows the thickness loss regarding development time. The sample had no PAG and quencher, resulting in the fastest etch rate of $>2 \mathrm{~nm} / \mathrm{s}$. The presence of PAG and quencher effectively reduced the etch rate to $0.5 \mathrm{~A} / \mathrm{s}$. PAG worked as a development inhibitor and reduced the etch rate as well as the backbone polymer, as reported previously [31], [32]. No distinct difference in term of etch rate was observed between the presence of $1.5 \mathrm{wt} \%$ quencher with PAG in PHS:tBA. Fig. 5(b) shows the RMS performance regarding development time. The sample with PHS:tBA-only shows no roughness response with the development time. The film was mostly removed in the first $10 \mathrm{~s}$ of development. The AFM measurement was most likely conducted on the silicon substrate but not on the photoresist. The samples with PAG and quencher and without quencher show a similar trend as the surface roughness increases with the development time. Fig. 6 shows the surface evolution with development time. The RMS increased from 0.29 $\mathrm{nm}$ after coating to $2.52 \mathrm{~nm}$ after $110 \mathrm{~s}$ of development.
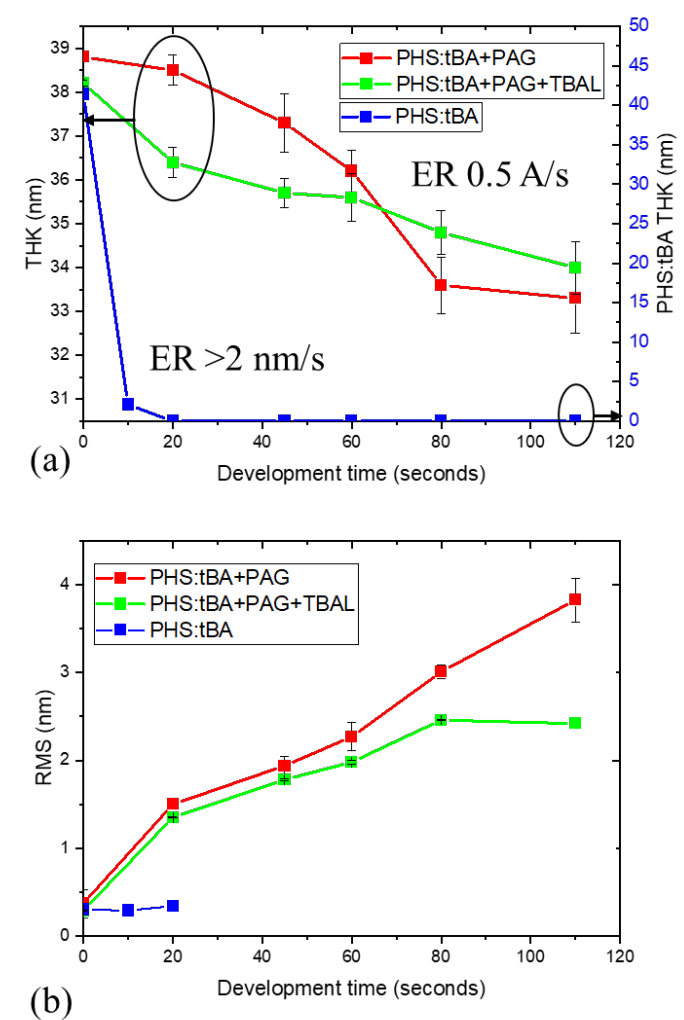

Fig. 5. (a) Film thickness change and (b) RMS change with the development time in the resist system of PHS:tBA

Fig. 7 (a) and (b) show an AFM line scan comparison between the development time and comparison of height histogram. The surface roughness or variation is clearly associated with the development time as well as "valley" formation. The "valley" is classified as a big trench on the resist surface. The implication of "valley" formation is 


\section{AFM images of PHS:TBA $83.5 w t \%$ + PAG $15 w t \%+$ TBAL $1.5 w t \%$}

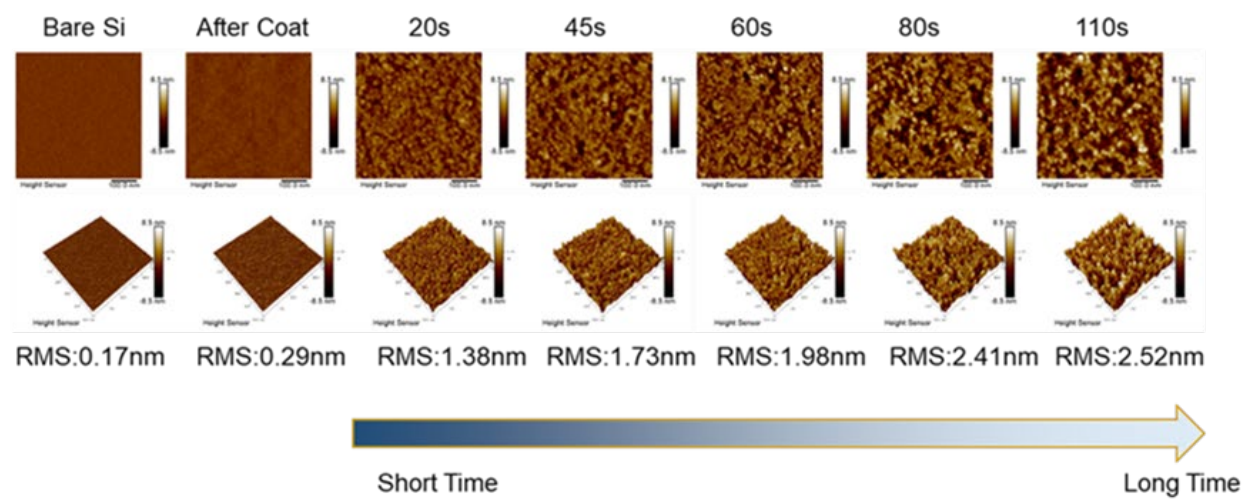

Fig. 6. AFM images of PHS:tBA with $15 \mathrm{wt} \%$ of PAG and $1.5 \mathrm{wt} \%$ of TBAL with the development time.

shown as a localized material loss with a relatively faster etch rate than the adjacent site and resulted in a rough surface. A similar result was observed in the height histogram. The histogram was started in the shape of a normal distribution in $0 \mathrm{~s}$ and then progressed into a skewness into the region of negative height. This indicates a localized dissolution rate difference that tends to be correlated with the resist component and inhomogeneity of resist structure.
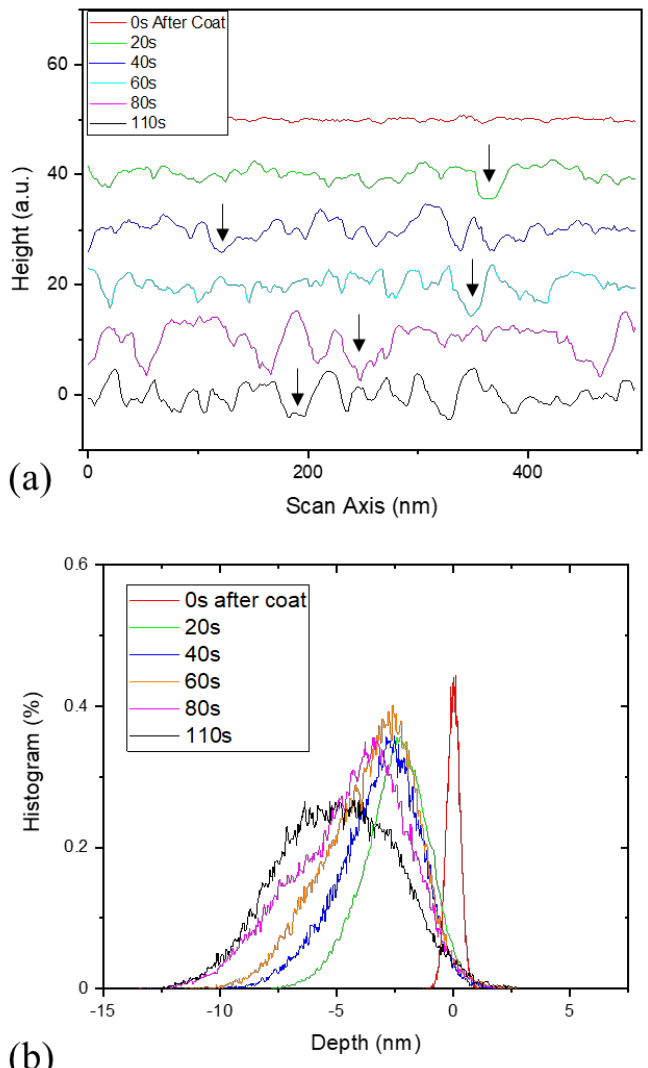

(b)

Fig 7. (a) Line scan and (b) height histogram of PHS:tBA with $15 \mathrm{wt} \%$ of $\mathrm{PAG}$ and $1.5 \mathrm{wt} \%$ of TBAL with the development time. The black arrows show examples of valley formation.
Fig. 8 shows the ratio of valley area to the total scan area with the development time. A clear valley area expansion was associated with the development time. In the first $20 \mathrm{~s}$, the valleys were created locally and accounted only $<10 \%$ of the total scan area. The area increased with the development time. At $80 \mathrm{~s}$ of development, the valleys account for close to $65 \%$ of the scan area of the resist and show a few features that protruded out of the surface. At $110 \mathrm{~s}$ of development, the valley area was slightly reduced to around $55 \%$ from the possible undercut etch and the isotropic etch behavior of the wet dissolution.

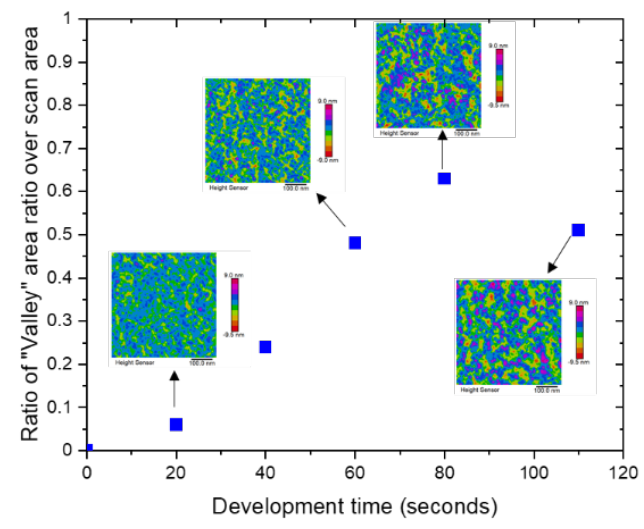

Fig 8. Ratio of "valley" area over the scan area with the development time.

3.3. Dark loss induced surface variation in PHSPS:tBA system and PAG loading

In this section, the role of backbone polymers and PAG loading to dark loss induced surface variation is discussed. Fig. 9 (a) shows the thickness loss of tested samples with the development time. The PHS:tBA sample with PAG and quencher had an etch rate of about $0.5 \mathrm{~A} / \mathrm{s}$. The other tested samples had an etch rate of $<0.1 \mathrm{~A} / \mathrm{s}$ or no average film loss. 
The development rate on these tested samples was effectively reduced by changing the polymer system from PHS:tBA to PHSPS:tBA. The presence of polystyrene in the copolymer system tends to alleviate the film loss during the wet process because it does not contain a polar group in the structure. One commercially available CAR resist was also included in the comparison with equivalent development condition. No noticeable thickness change was observed in this resist.
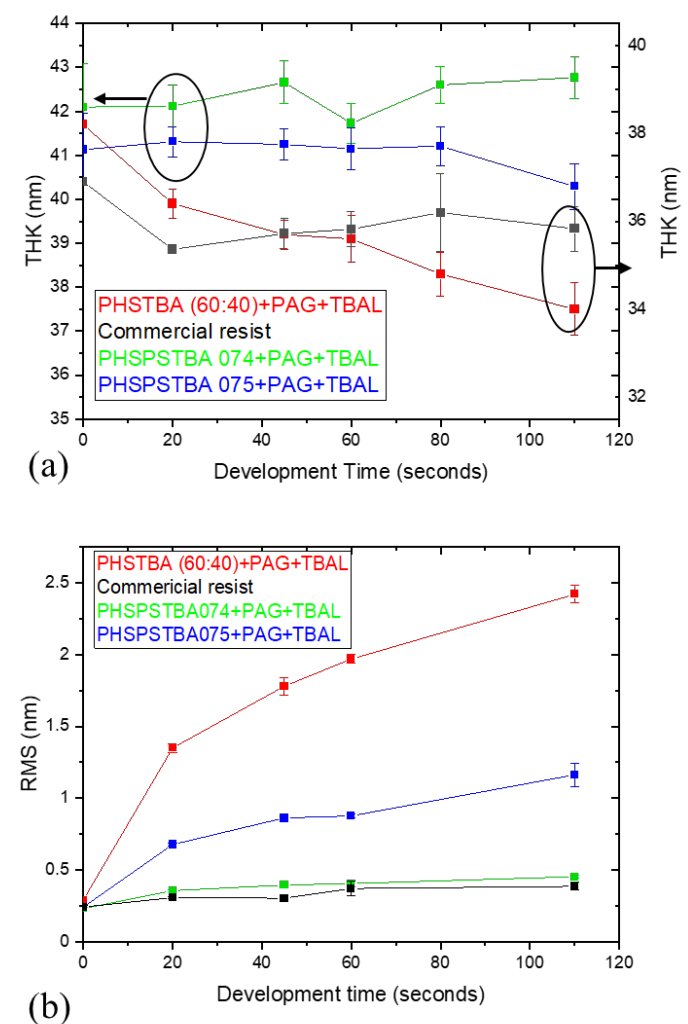

Fig. 9. (a) Film thickness change and (b) RMS change with the development time.

Fig. 9 (b) shows the progression of surface roughens with development time in PHS:tBA, PHSPS:tBA 074, PHSPS:tBA 075, and the commercial resist. The general trend was observed as the surface roughness increased with the development time among all the tested samples. The reduction in film loss in the presence of polystyrene can also be attributed to a reduction in the degradation of surface roughness. The amount of polystyrene in the two PHSPS:tBA systems also significantly affected the surface roughness. PHSPS:tBA 074 has $24 \%$ of polystyrene than $19 \%$ in 075 in the copolymer system, resulting in an RMS difference from $0.4 \mathrm{~nm}$ to $1.01 \mathrm{~nm}$ under $110 \mathrm{~s}$ of development process. The presence of PAG and polystyrene works as a dissolution inhibitor, corresponding to a slower etch rate in the development process than the PHS:tBA system. The etch selectivity between the components of the resist is clearly associated with the dark loss induced surface roughness. In the PHSPS:tBA system, both components of backbone polymer and PAG had a relatively slower etch rate, thus resulting in a minor degradation of surface roughness with the development process. In the PHS:tBA system, the backbone polymer and PAG had a significant difference in etch rate. The faster etch rate component tends to be removed first, and the slow etch rate components tend to remain on the surface for creating a rough surface. The commercially available resist had a similar surface roughness as PHSPS:tBA 074 system with minor dark loss induced surface roughness.
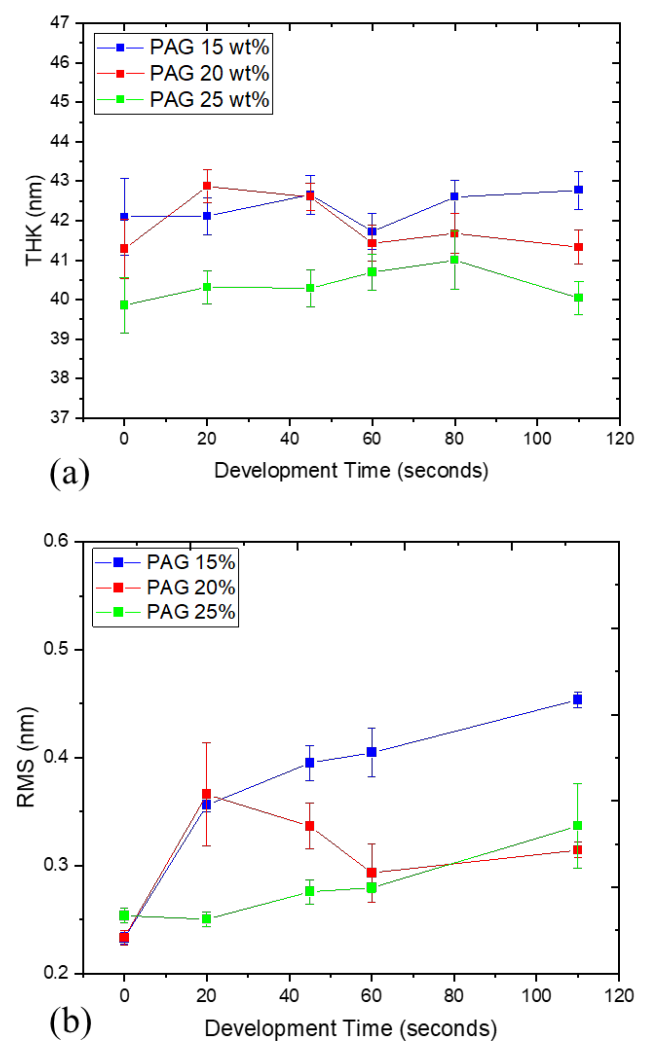

Fig. 10. PAG loading to (a) film thickness change and (b) RMS change with the development time in the resist system of PHSPS:tBA 074 with TBAL.

Fig. 10 (a) shows the film loss with development time in the PHSPS:tBA 074 resist with $1.5 \mathrm{wt} \%$ of TBAL and PAG loading of 15,20 , and $25 \mathrm{wt} \%$. Similar results are also observed in Fig. 9 (a). No obvious film loss was observed with the increase of 
development time. The change in film thickness was within the sample variation. Fig. 10 (b) shows the RMS performance with the development time between the PAG loading tested conditions. In general, the surface roughness increased with development time. The high concentration of PAG has the lowest RMS. PAG was added to reduce the etch selectivity between resist components. Fig. 11 shows a PSD comparison between the PAG loading tested conditions. The tested condition with $15 \mathrm{wt} \%$ of PAG had a high PSD within the most frequency domain. The correlation length beyond $100 \mathrm{~nm}$ was not considered due the lack of data points within the limited scan area. By increasing the PAG loading from $15 \mathrm{wt} \%$ to $25 \mathrm{wt} \%$, a change in correlation length in spatial frequency was observed. The correlation length increased from $33 \mathrm{~nm}$ to close to $100 \mathrm{~nm}$. The presence of PAG affects both average surface roughness and the spatial frequency of surface topography from the PSD observation.

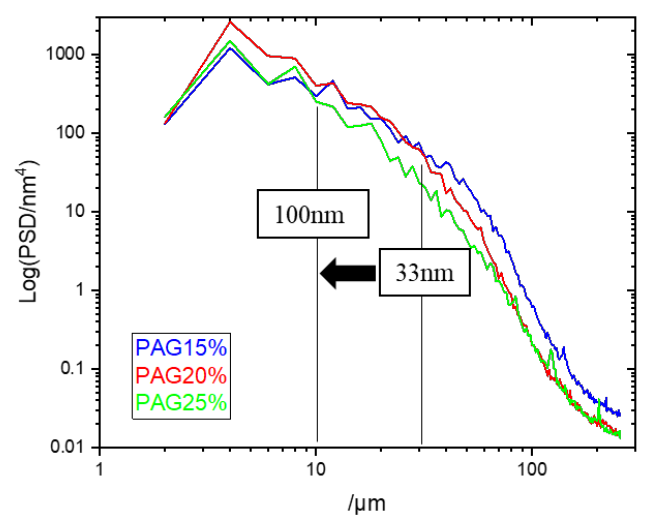

Fig. 11. PSD comparison of PAG loading tested conditions in the resist system of PHSPS:tBA 074 and TBAL at development of $45 \mathrm{~s}$.

\section{Summary and Conclusion}

In summary, the surface topography of an EUV photoresist was characterized by AFM. In a particular CAR system, the photochemistry and dark loss were the main contributors to the overall surface variation. An extensive study was conducted to evaluate the effect of dark loss including the backbone polymer system and PAG loading. The AFM data was processed and extracted to determine the height histogram, line scan, area ratio calculation, and PSD. Form this information, the dark loss induced surface roughness can be attributed to the competition of etch selectivity in resist components. When the etch selectivity is relatively low as well as low film loss, the dark loss induced surface roughness remains relatively smooth. In the case of a high etch selectivity between the components, a substantial difference in etch rate in the deprotection front led to a high surface roughness as well as a change of correlation length in PSD and skewness in the height histogram.

\section{Acknowledgement}

This work was funded in part by Semiconductor Research Corporation (SRC) Nanomanufacturing and Processes (NMP) research program, and it was performed at the State University of New York Polytechnic Institute, College of Nanoscale Science and Engineering at Albany, NY. We would like to thank Sandra Schujman for her in-depth knowledge and valuable help in the AFM measurements.

\section{References}

1. H. J. Levinson and T. A. Brunner, Proc. SPIE, 10809 (2018) 1080903.

2. P. De Bisschop, J. Micro/Nanolithography, MEMS, MOEMS, 16 (2017) 1.

3. P. P. Naulleau, C. N. Anderson, L.-M. Bacleaan, P. Denham, S. George, K. A. Goldberg, G. Jones, B. McClinton, R. Miyakawa, S. Rekawa, and N. Smith, Proc. SPIE, 7972 (2011) 797202.

4. S. Bhattarai, A.R. Neureuther, and P. P. Naulleau, J. Vac. Sci. Technol. B, Nanotechnol. Microelectron. Mater. Process. Meas. Phenom., 35 (2017) 061602.

5. C. A. Mack, Proc. SPIE, 11147 (2019) 111470A.

6. J. W. Thackeray, Proc. SPIE, 7972 (2011) 797204.

7. L. Meli, K. Petrillo, A. De Silva, J. Arnold, N. M. Felix, C. Robinson, B. Briggs, Y. Mignot, J. Shearer, B. Hamieh, K. Hontake, L. Huli, C. Lemley, D. Hetzer, T. Shimoaoki, Y. Hashimoto, H. Ichinomiya, A. Kai, S. Kawakami, K. Tanaka, A. Jain, B. Saville, C. Lenox, S. Matham, E. Liu, K. Akiteru, and H. Choi, Proc. SPIE, 10583 (2018) 105830E.

8. Raley, C.A. Mack, E. Liu, S. Thibaut, and A. Ko, Proc. SPIE, 10809 (2018) 1080915.

9. Ashim Dutta, J. Church, J. Lee, L. Meli, C. Chun Liu, S. Sharma, K. Petrillo, C. Murray, E. Liu, K. Lutker-Lee, Q. Lou, C. Cole, A. Raley, A. Ko, S. Kal, J. Kaminsky, A. Mosden, H. Zhang, S. Hu, L. Huli, N. Shibata, D. Hetzer, and C.-Y. Hsieh, Proc. SPIE, 11323 (2020) $113230 \mathrm{~V}$.

10. E. Liu, S. Thibaut, A. Ko, and P. Biolsi, Proc. SPIE, 10963 (2019) 109630R.

11. A. H. Gabor, A. Brendler, T. A. Brunner, X. 
Chen, J. Culp, and H. Levinson, Proc. SPIE, 10583 (2018) 105830C.

12. D. De Simone, V. Rutigliani, G. Lorusso, P. De Bisschop, Y. Vesters, V. M. Blanco Carballo, and G. Vandenberghe, Proc. SPIE, 10583 (2018) 105830G.

13. E. Liu, A. Ko, R.A. Farrell, D. O'Meara, C.-Y. Hsieh, and P. Biolsi, Proc. SPIE, 10584 (2018) 1058408.

14. D. Nagy, G. Indalecio, A. J. Garcia-Loureiro, M.A. Elmessary, K. Kalna, and N. Seoane, IEEE J. Electron Devices Soc., 6 (2018) 332.

15. Y. Luo and P. Gupta, Proc. SPIE, 10588 (2018) 1058800 .

16. Robinson and R. Lawson, Materials and Processes for Next Generation Lithography (2016).

17. K. Nakamura, "Photopolymers: Photoresist Materials, Processes, and Applications" (2014).

18. U. Okoroanyanwu, Chemistry and Lithography (SPIE, 2010).

19. M. Toriumi, K. Kaneyama, S. Kobayashi, and T. Itani, Proc. SPIE, 6923 (2008) 69230L.

20. T. Yoshimura, H. Shiraishi, J. Yamamoto, and S. Okazaki, Appl. Phys. Lett., 63 (1993) 764.

21. C. A. Cutler, J. F. Mackevich, J. Li, D. J. O'Connell, G. F. Cardinale, and R. L. Brainard, Proc. SPIE, 5037 (2003) 406.

22. T. Yoshimura, H. Shiraishi, J. Yamamoto, and S. Okazaki, Jpn. J. Appl. Phys., 32 (1993) 6065.
23. N. Kubota, T. Hayashi, T. Iwai, H. Komano, and A. Kawai, J. Photopolym. Sci. Technol., 16 (2003) 467.

24. I. U. Ahad, M. A. Obeidi, B. Budner, A. Bartnik, H. Fiedorowicz, and D. Brabazon, AIP Conf. Proc., 1896 (2017) 200008.

25. B. C. Park, Y. J. Cho, I. Kim, and J. Yeo, Proc. SPIE, 8681 (2013) 86812K.

26. D. Schmidt, K. Petrillo, M.A. Breton, J. Fullam, S. Hand, J. Osborne, W. Wang, and D. Fey, Proc. SPIE, 11325 (2020) 113250T.

27. C. Mbanaso, S. Kruger, C. Higgins, Y. Khopkar, A. Antohe, B. Cardineau, and G. Denbeaux, Proc. SPIE, 7969 (2011) 79692J.

28. S. Grzeskowiak, A. Narasimhan, E. Rebeyev, S. Joshi, R. L. Brainard, and G. Denbeaux, $J$. Photopolym. Sci. Technol., 29 (2016) 453.

29. Narasimhan, S. Grzeskowiak, B. Srivats, H. Herbol, L. Wisehart, C. Kelly, W. Earley, L.E. Ocola, M. Neisser, G. Denbeaux, and R.L. Brainard, Proc. SPIE, 9422 (2015) 942208.

30. S. Grzeskowiak, A. Narasimhan, M. Murphy, L. Napolitano, D. A. Freedman, R. L. Brainard, and G. Denbeaux, Proc. SPIE, 10146 (2017) 1014605.

31. B. Kumar and D.L. Goldfarb, Proc. SPIE, 10586 (2018) 1058604.

32. H. Ito, D.-F. Alexander, and G. Breyta, J. Photopolym. Sci. Technol. 10 (1997) 397. 